\title{
Nanomaterials for Light Management in Electro-Optical Devices
}

\author{
Vo-Van Truong, ${ }^{1}$ Jai Singh, ${ }^{2}$ Sakae Tanemura, ${ }^{3}$ and Michael $\mathrm{Hu}^{4}$ \\ ${ }^{1}$ Department of Physics, Concordia University, Montréal, QC, Canada H4B 1R6 \\ ${ }^{2}$ Faculty of Engineering, Health, Science and the Environment, Charles Darwin University, Darwin, NT 0810, Australia \\ ${ }^{3}$ Japan Fine Ceramics Center, Nagoya 456-8587, Japan \\ ${ }^{4}$ Energy and Transportation Science Division, Oak Ridge National Laboratory, Oak Ridge, TN 37831, USA
}

Correspondence should be addressed to Vo-Van Truong, tvovan@alcor.concordia.ca

Received 26 August 2012; Accepted 26 August 2012

Copyright ( 2012 Vo-Van Truong et al. This is an open access article distributed under the Creative Commons Attribution License, which permits unrestricted use, distribution, and reproduction in any medium, provided the original work is properly cited.

In the past decade, nanostructured materials and nanoparticles have emerged as the necessary ingredients for electrooptical applications and enhancement of device performance, in particular by making use of the light management aspects of the nanomaterials. The application areas that are being transformed profoundly include smart coating devices (e.g., electrochromic, photochromic, and thermochromic devices), solar energy, and sensing. Despite the large volume of work in the past on smart coating devices, and in particular on electrochromic devices and thermochromic fenestrations, for optical transmission or reflection control, applications remain limited because of slow response time and nonuniformity in the case of large surfaces. Recent works in the field indicate that nanostructured electrochromic coatings would be an integral part of the solution to the above problem. One aspect that can thus be focused on would be the fabrication and characterization of the nanostructured smart coating materials and their compatibility with other layers in the overall smart coating device. In the area of solar photovoltaics, nanomaterials have been used in designing light-trapping schemes for inorganic as well as organic solar cells. One particular category of solar cells that has attracted much interest is the plasmonic solar cells in which metallic nanoparticles are incorporated, helping in enhancing their energy conversion efficiency. Nanostructured solar cells would eventually develop into a "game changing" technology for making solar cells that are affordable and highly efficient, providing a sizeable alternative energy source for our everincreasing energy needs. Sensors based on the optical properties of constituting nanostructures and nanoparticles also form a most interesting class of bio- and electrochemical sensing devices. The possibility of synthetizing nanoparticles and structures of specifically desired sizes and shapes has indeed opened a whole new range of sensing applications. In parallel to the experimental development of nanomaterials for light management in devices, theoretical modeling and analysis have also accomplished much progress, and different methods for simulating the optical properties of nanoparticles and structures have been proposed. This special issue of the Journal of Nanomaterials is thus dedicated to articles dealing with nanostructured materials that can be used for light management purpose in different applications.

Silicon-rich oxide (SRO) is a dielectric material that contains Si nanoparticles and exhibits interesting physical characteristics for applications in optoelectronic devices. The work by Aceves-Mijares et al. examine, in detail the electro-, cathode-and photoluminescence properties of SRO and discuss the origin of light emission in this type of materials. SRO films, of high and medium silicon excess density, obtained by low-pressure chemical vapor deposition and annealed at $1,100^{\circ} \mathrm{C}$ have been studied. Results obtained by the authors have led to conclude that SRO emission properties are due to oxidation state nanoagglomerates rather than to nanocrystals, and the emission mechanism is similar to that in the donor-acceptor decay in semiconductors with a specific wide emission spectrum.

Two papers are devoted to nanostructured electrochromic thin films, a category of materials most suitable for controlling light transmission or absorption in electrooptical devices, including smart window coatings. Dinh et al. have shown that by mixing nanostructured $\mathrm{Ti}$ and $\mathrm{W}$ oxides 
films, one can obtain devices with considerable enhancement of electrochromic efficiency and electrochemical stability as compared to the conventional nonnanostructured films. As large-area mixed $\mathrm{Ti}$ and $\mathrm{W}$ oxides can be prepared by the simple doctor blade technique followed by an electrochemical process, this type of nanostructured electrochromic films can be considered a good candidate for smart window applications. In another paper, Djaoued et al. have presented their studies on the synthesis, characterization, and electrochromic applications of porous $\mathrm{WO}_{3}$ thin films with different nanocrystalline phases. Asymmetric type electrochromic devices have been constructed using hexagonal, monoclinic, and orthorhombic porous $\mathrm{WO}_{3}$ thin porous films, and their enhanced electrochromic functionality has been well demonstrated. The paper on smart materials by Chen et al. presents the synthesis of thermochromic Wdoped $\mathrm{VO}_{2}$ (monoclinic/rutile) nanopowders using a novel and simple solution-based process as opposed to other conventional techniques such as excimer laser-assisted metal organic deposition and magnetron sputtering. This simple process is based on the reaction of ammonium metavanadate $\left(\mathrm{NH}_{4} \mathrm{VO}_{3}\right)$ and oxalic acid dihydrate $\left(\mathrm{C}_{2} \mathrm{H}_{2} \mathrm{O}_{4} \cdot 2 \mathrm{H}_{2} \mathrm{O}\right)$ followed by addition of appropriate ammonium tungstate $\left(\mathrm{N}_{5} \mathrm{H}_{37} \mathrm{~W}_{6} \mathrm{O}_{24} \cdot \mathrm{H}_{2} \mathrm{O}\right)$.

$\mathrm{ZnO}$ nanorods photoluminescence properties are reported by E. Sönmez and K. Meral. The authors have investigated the correlation of photoluminescence lifetime between $\mathrm{ZnO}$ nanorods and thiourea-doped $\mathrm{ZnO}$ nanorods and concluded that by making use of thiourea, the photoluminescence lifetime of $\mathrm{ZnO}$ nanorods can be enhanced significantly. The structural changes observed in thiourea-containing samples are reflected in thinner and longer $\mathrm{ZnO}$ nanorods as compared to those without thiourea. $\mathrm{Xi}$ et al. have synthesized single-crystal silicon oxynitride nanowires with high $\mathrm{N}$ concentrations on silicon substrate at $1200^{\circ} \mathrm{C}$ without any metal catalyst. This process has yielded ultrabright white nanowires that can be used as reflecting surfaces in bright white light-emitting-diodes for lighting applications. A mechanism for the growth of these typical nanowires is also proposed by these authors. Banerjee et al. in their paper have studied the photocatalytic degradation of organic dyes by sol gel-derived gallium-doped anatase titanium oxide. With these doped nanoparticles, almost 90\% degradation efficiency can be achieved within 3 hrs of UV irradiation of organic dye samples. It is suggested that the cost-effective $\mathrm{TiO}_{2}$ : Ga nanoparticles can be used efficiently for lightassisted oxidation of toxic organic molecules.

A final contribution is made by S. Gauvin and $\mathrm{M}$. Boudreau in the field of nonlinear multilayer microcavities. The authors have shown that even in the case of very weak pump-depletion conditions, an optical structure that allows multipass optical paths, such as high finesse multilayer microcavities, could lead to significant cumulative pump wave depletion. To account for these nonnegligible effects, the authors have developed a matrix formalism that can be applied to absorbing media and extended to include anisotropic layers and cascading effects.

\section{Acknowledgments}

The editors would like to express their gratitude to authors as well as reviewers for their contributions and efforts in participating in the production of this special issue.

Vo-Van Truong Jai Singh

Sakae Tanemura

Michael Hu 

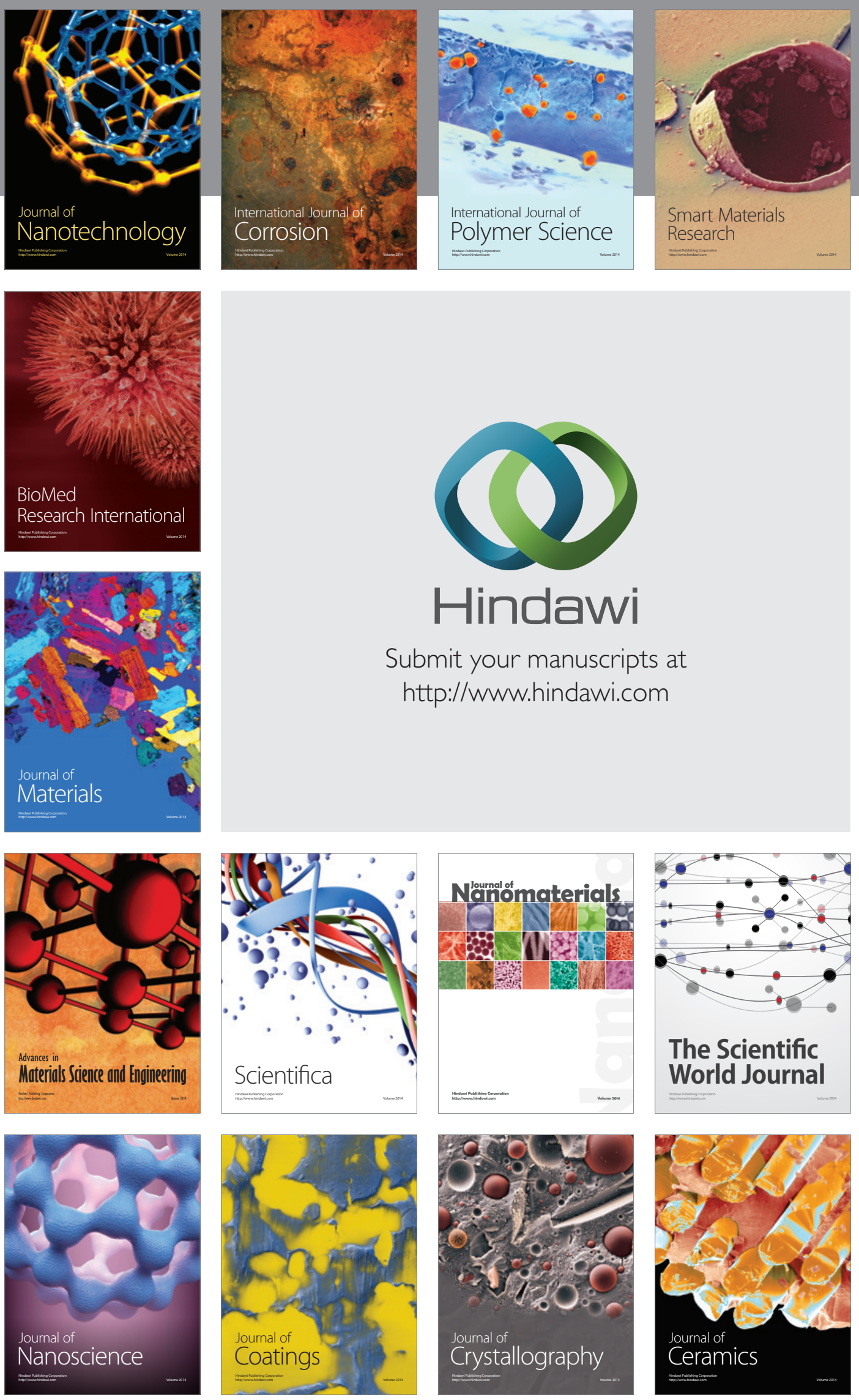

The Scientific World Journal

Submit your manuscripts at

http://www.hindawi.com

\section{World Journal}

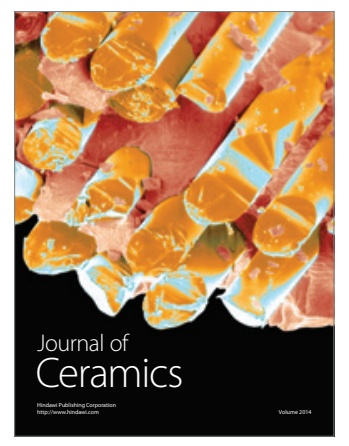

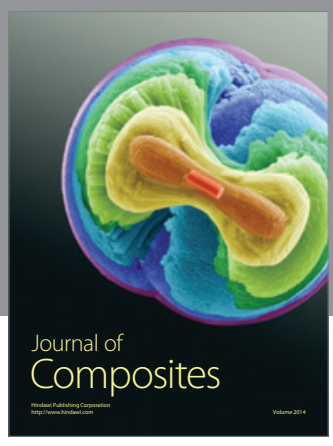
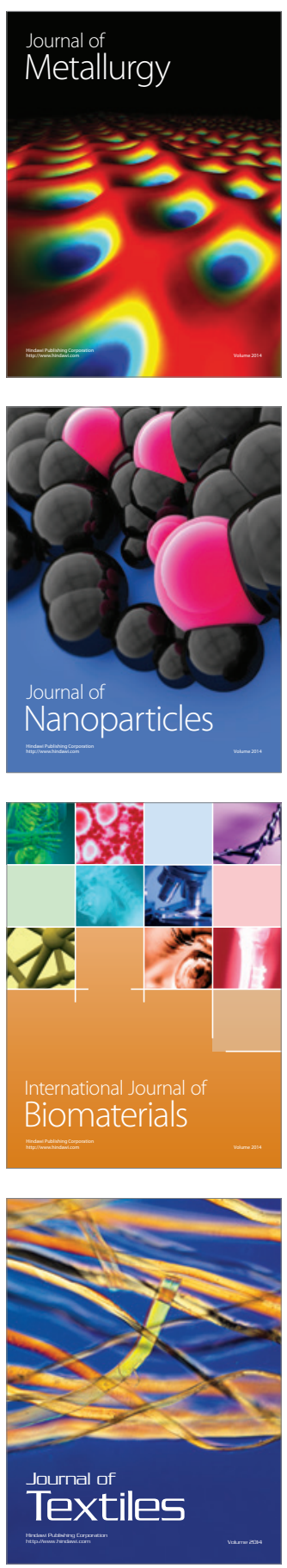